\title{
L'apport et les perspectives du génie génétique et des biotechnologies
}

Les techniques des anticorps monoclonaux et des recombinants d'ADN sont à l'origine d'une remarquablenévolution des stratégies industrielles dans le domaine de la santé, qu'il s'agisse du développement de nouveaux procédés diagnostiques, de nouveaux vaccins ou de la production de nouveaux médicaments.

\section{Terence Cartwright \\ Directeur de recherche biotech- nologique}

T. Cartwright: Recherche biotechnologique Rhône-Poulenc Santé, Centre de Recherche de Monts, 18 rue de Montbazon, 37260 Monts,

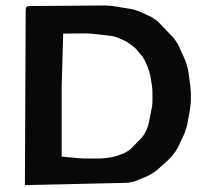

ans les années 1970, l'industrie pharmaceutique a connu l'émergence d'un phénomène nouveau, d'une importance considérable et grandissante. Des biotechnologies nouvelles sont apparues, initialement dans des laboratoires universitaires puis, très rapidement, dans un ensemble de petites sociétés à capital risque. Différentes entreprises pharmaceutiques ont répondu à cette nouvelle potentialité soit en créant leurs propres unités de biotechnologie, soit en établissant des collaborations étroites avec des sociétés spécialisées. Quoique des approches biotechnologiques aient été utilisées depuis longtemps par l'industrie, notamment pour la production, par fermentation, d'antibiotiques et autres produits, il est vite apparu que cette utilisation nouvelle de la biotechnologie pourrait ouvrir des horizons nouveaux pour le développement de nouveaux médicaments et pourrait changer de façon fondamentale les méthodes de production de certains produits existants. Son impact sur l'industrie pharmaceutique, et donc sur le développement futur des domaines santé, sera très important ; il a été estimé que, pour l'année 1990, plus de huit milliards de dollars de vente seront engendrés par des produits pharmaceutiques dérivés des biotechnologies [1].

Bien que la liste des produits obtenus par cette technologie, et disponibles aujourd'hui pour une utilisation clinique, soit encore relativement peu importante (Tableau I), de nombreuses substances sont actuellement en cours de développement dans l'industrie pharmaceutique.

L'objectif de cet article est de considérer certains de ces développements et leur apport éventuel dans le domaine de la santé.

\section{Une activité pluridisciplinaire}

Deux approches technologiques récentes sont au cœur même de cette nouvelle biotechnologie. La première est le génie génétique, ou technologie de l'ADN recombinant, qui permet, dans un organisme hôte génétiquement modifié, la production de quantités de protéines et de peptides non accessibles auparavant. Ces protéines peuvent être des enzymes, des récepteurs, des hormones, des peptides régulateurs, des facteurs de croissance, des immunomodulateurs, etc. En tant que telles, elles représentent au niveau moléculaire des éléments directement impliqués dans le contrôle de l'homéostasie et dans la biologie des états pathologiques. 
L'autre avancée technologique majeure est la capacité de produire des anticorps monoclonaux qui, possédant une spécificité parfaitement définie, sont capables de former des complexes antigènes-anticorps avec des épitopes précisément définis sur les protéines ou autres molécules.

Néanmoins, il ne faut pas oublier que, à côté de ces deux « vedettes » de la nouvelle biotechnologie, des avancées majeures dans les domaines de la biochimie, la chimie organique, la physique et l'informatique ont toutes contribué au développement d'un extraordinaire progrès de la biotechnologie. Plus spécifiquement, les progrès dans la synthèse des nucléotides et des peptides, le développement des systèmes de chromatographie liquide en haute pression (CLHP) pour la séparation des protéines, les techniques de séquençage de protéines et des acides nucléiques et les performances du graphisme moléculaire, sont tous des éléments clés dans la réalisation d'une nouvelle génération de produits d'origine biotechnologique dans le domaine de la santé.

\section{Un impact très large dans le domaine santé}

Les produits de l'industrie santé peuvent être raisonnablement divisés en trois groupes: les produits diagnostiques, les produits prophylactiques et les produits thérapeutiques. La biotechnologie a un impact potentiel dans ces trois domaines, impact déjà concrétisée dans les deux premiers, pour lesquels les barrières réglementaires sont plus faciles à franchir.

Produits diagnostiques. Il est déjà évident que l'apparition sur le marché des anticorps monoclonaux absolument spécifiques et reproductibles a permis d'étendre et d'améliorer les procédés immunodiagnostiques. En conséquence, il y a eu une multiplication importante des trousses de diagnostic immunologiques (kits), y compris celles destinés à $\mathrm{m} / \mathrm{s} n^{\circ} 4$ vol. 4, auril 88
Tableau I

PRODUITS PHARMACEUTIQUES D'ORIGINE BIOTECHNOLOGIQUE ACCEPTÉS PAR LE FDA* SUR LE MARCHÉ AUX ÉTATS-UNIS

- Insuline

- Hormone de croissance humaine

- Interféron

- Vaccin contre I'hépatite B

- Activateur tissulaire du plasminogène (tPA)**

* FDA : Food and drug administration. * Produit Genentech accepté par le FDA le 13 novembre 1987.

une utilisation privée. Le développement récent des anticorps monoclonaux hybrides [2], portant, sur la même molécule d'anticorps, deux sites de reconnaissance pour des épitopes différents, conféra aux procédés immunodiagnostiques une puissance et une sensibilité accrues.

In vivo, la spécificité absolue des anticorps monoclonaux peut assurer leur fixation uniquement sur les cellules qui portent l'épitope correspondant. Cette capacité est utilisée à des fins diagnostiques en employant des anticorps monoclonaux marqués pour la détection et la localisation dans l'organisme d'un type cellulaire donné (identification ou imagerie). La même approche peut être utilisée à des fins thérapeutiques quand un médicament est couplé à l'anticorps, favori- sant ainsi son transport vers des cibles cellulaires specifiques, par exemple les cellules tumorales (ciblage).

Un autre secteur du diagnostic dans lequel les fruits des avances en biotechnologie sont déjà exploités est l'utilisation de sondes nucléotidiques pour la détection d'anomalies génétiques. Plusieurs maladies d'importance socio-économique majeure peuvent être détectees en période prénatale par hybridation moléculaire appliquée à des cellules fotales (Tableau II). Les techniques employées deviennent aujourd'hui suffisamment simples pour permettre la détection des porteurs d'anomalies telles que par exemple l'anémie falciforme, la thalassémie ou la mucoviscidose. Un traitement primaire n'est pas encore possible pour les maladies génétiques. Cependant, le coût des soins de ces trois maladies graves suggère qu'un programme de détection précoce systématique aurait un impact important dans le domaine de la santé [3].

La technologie d'hybridation moléculaire peut également être appliquée à la détection dans les tissus ou les sécrétions des agents infectieux tels que les virus, les bactéries et les parasites. Un avantage de cette approche par rapport au diagnostic immunologique est que l'hybridation moléculaire peut détecter l'agent même si celui-ci n'exprime pas des antigènes détectables. Cette capacité

\begin{tabular}{|c|c|}
\hline $\begin{array}{r}\text { EXEMPLES DE } \\
\text { PAR }\end{array}$ & $\begin{array}{l}\text { II } \\
\text { NÉTIQUES DÉTECTABLES } \\
\text { UCLÉOTIDIQUES }\end{array}$ \\
\hline Maladies & $\begin{array}{c}\text { Nombre de cas sur } \\
45000 \text { naissances (en Europe) }\end{array}$ \\
\hline $\begin{array}{l}\beta \text {-thalassémie } \\
\text { Anémie falciforme } \\
\text { Myopathie de Duchenne } \\
\text { Mucoviscidose } \\
\text { Hémophilie } \\
\text { Chorée de Huntington }\end{array}$ & $\begin{array}{c}5 \text { à } 200^{*} \\
\simeq 2 \\
4-6 \\
22 \\
3 \\
\simeq 5\end{array}$ \\
\hline
\end{tabular}


est particulièrement importante dans le cas de virus qui peuvent être présents sous forme latente (SIDA, hépatite $B$, virus de papillome, etc.) ou dans le cas des parasites qui expriment des antigènes différents selon le stade de leur cycle (trypanosomiases, paludisme, etc.).

\section{Produits prophylactiques}

La prévention des maladies infectieuses par vaccination est un des accomplissements majeurs de la science médicale. Cependant, tous les vaccins couramment disponibles ne sont pas idéaux dans les domaines de la sécurité, de l'efficacité et du prix. De plus, pour certaines maladies, telles que l'hépatite virale, le paludisme et le SIDA, les méthodes «classiques » de production de vaccin ne sont pas satisfaisantes du fait des difficultés d'isolement en quantité suffisante d'un antigène efficace et de caractère immunologique constant.

La possibilité de produire, par génie génétique, une protéine donnée en grande quantité et sous une forme hautement purifiée a donc trouvé une application immédiate dans l'élaboration des vaccins. Plus récemment, la capacité de produire des segments sélectionnés de ces protéines, fusionnés avec d'autres protéines ou assemblés dans des particules de façon à créer une antigénicité améliorée, a permis pour la première fois la production de vaccins de bonne qualité, efficaces contre des agents pathogènes difficiles à cultiver tels que le virus de l'hépatite $B$. En outre, la pureté de l'antigène diminue le risque d'apparition d'effets secondaires provoqués par le vaccin. D'autre part, l'absence totale d'agent pathogène intact pendant sa production élimine toute possibilité de transmission d'un agent infectieux comme cela est à craindre dans le cas des vaccins mal inactivés.

Bien que des vaccins ne soient pas encore disponibles contre le SIDA ou le paludisme, des progrès

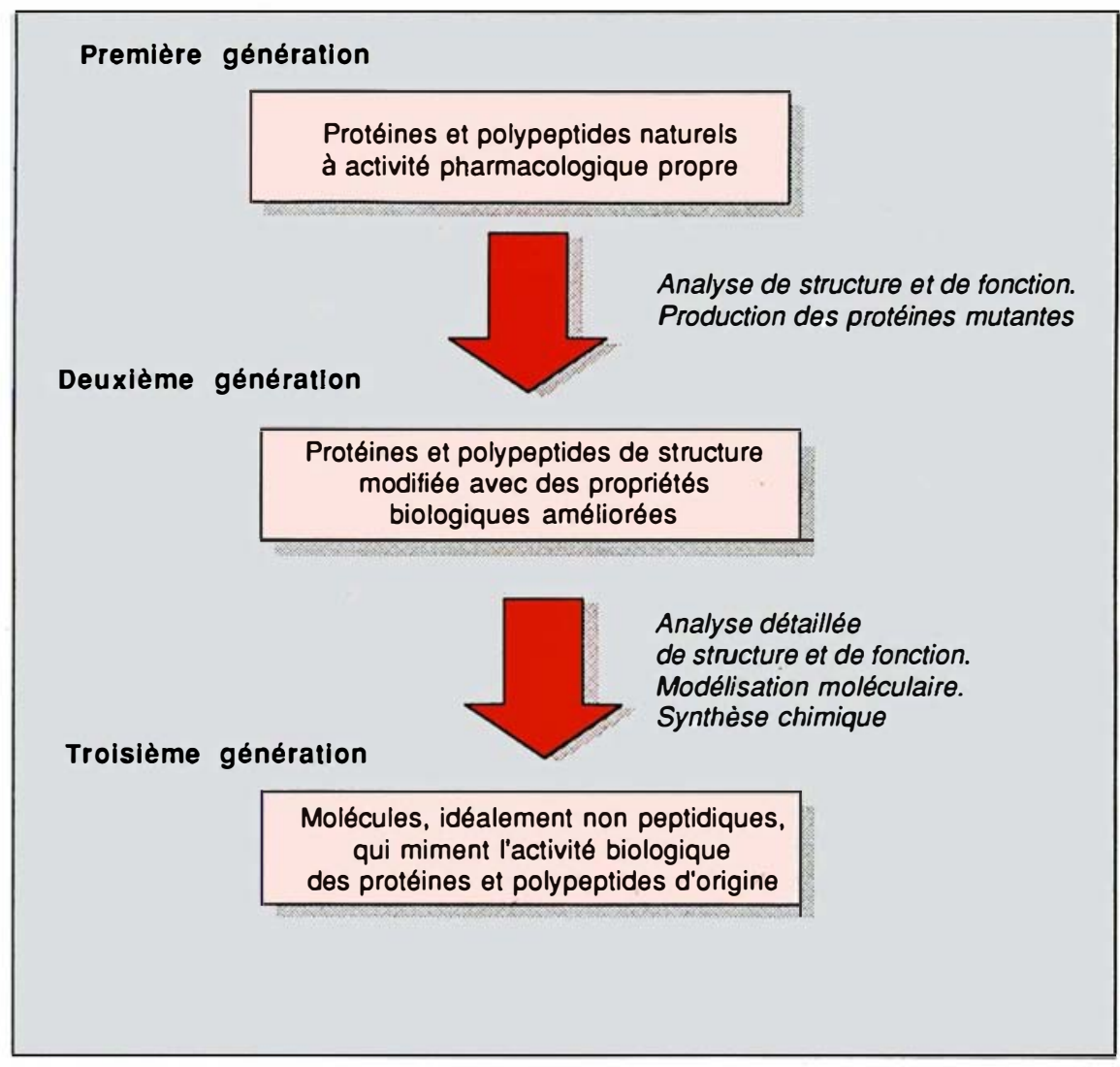

Figure 1. Les trois "générations" des produits dérivés des protéines et des polypeptides recombinants.

technologie est en première ligne dans cette lutte. L'apport direct de la biotechnologie au développement d'un de ces vaccins recombinants, le vaccin contre l'hépatite $\mathrm{B}$, sera discuté en détail dans ce numéro, dans l'article du professeur Tiollais (page 249).

Produits à usages pharmacologique et thérapeutique. La pharmacologie est la science de l'interaction entre des drogues et des cibles dans un système biologique. Son application à la médecine est probablement le domaine où les biotechnologies et particulièrement la technologie du clonage génétique ont ouvert le plus grand nombre de nouveaux horizons. Beaucoup de molécules cibles ou régulatrices, cliniquement importantes, sont des protéines ou des peptides dérivés de précurseurs protéiques, qui peuvent exister à des concentrations extrêmement faibles in vivo. Le génie génétique, associé au progrès de la chi- mie des protéines, a permis l'expression de telles molécules dans des organismes recombinants et, pour la première fois, leur étude, à l'état pur et en quantité non limitée, est devenue possible.

Auparavant, l'utilisation pharmaceutique des protéines était restreinte à celles qui pouvaient être aisément extraites à partir des tissus ou des sécrétions d'animaux. Ce furent le cas de l'insuline, de l'hormone de croissance isolée à partir des glandes pituitaires de cadavres, de l'urokinase extraite à partir de l'urine, ou des produits du sang tels que l'albumine, le facteur VIII ou les immunoglobulines. Dans la plupart des cas, la production est limitée et l'emploi non exempt de risques. L'insuline animale peut provoquer des réponses immunitaires, la production d'hormone de croissance est sévèrement limitée par la disponibilité de tissus humains et celle d'urokinase 
demande la collecte de centaines de mètres cubes d'urine. Dernièrement la présence possible du virus du SIDA dans le sang est devenue une préoccupation majeure, ce qui est aussi vrai pour la contamination potentielle de l'hormone de croissance d'origine pituitaire par le virus de la maladie de Creutzfeldt-Jakob. La plupart de ces protéines peuvent maintenant être produites par les techniques de génie génétique, en grande quantité et sans aucun risque de contamination virale. D'autres protéines, dont l'intérêt clinique potentiel est évident, sont également maintenant disponibles, par exemple les interfé- rons $\alpha, \beta$ et $\gamma$, les interleukines 1 et 2 , l' $\alpha$ l-antitrypsine et l'activateur tissulaire du plasminogène (tPA).

\section{Développement des molécules obtenues par génie génétique}

Il convient de classer les produits de cette nouvelle approche pharmacologique en plusieurs phases ou générations (figure 1). Les produits de la première génération peuvent être considérés comme des protéines naturelles possédant une activité pharmacologique. Produits de première génération. Plusieurs produits de première génération ont déjà été mis sur le marché (Tableau I) et beaucoup d'autres sont à des étapes avancées d'essais cliniques. Une liste exhaustive de ces produits serait trop longue mais le phénomène est illustré pour le domaine cardiovasculaire, dans le Tableau III. Le médicament cardiovasculaire potentiellement le plus important est, sans aucun doute, le tPA, un agent thrombolytique enzymatique qui fait l'objet d'un article du professeur Samama dans ce numéro (page 222). Contrairement à d'autres enzymes thrombolytiques couramment utilisées (streptokinase et urokinase), l'activité du tPA dépend de la pré-

\begin{tabular}{|c|c|c|c|}
\hline \multicolumn{4}{|c|}{ Tableau III } \\
\hline Produit & Société & $\begin{array}{l}\text { Partenaire } \\
\text { industriel }\end{array}$ & $\begin{array}{l}\text { Stade des essais } \\
\text { cliniques }\end{array}$ \\
\hline tPA* & $\begin{array}{l}\text { Biogen } \\
\text { Celltech } \\
\text { Ciba Geigy } \\
\text { Chiron } \\
\text { Damon Biotech } \\
\text { Bio-Response } \\
\text { Genentech } \\
\\
\text { Genetics Institute } \\
\text { Integrated Genetics }\end{array}$ & $\begin{array}{l}\text { Smith Kline Beckman } \\
\text { Sankyo } \\
\text { Hoechst } \\
\text { Boehringer Ingelheim*** } \\
\text { Mitsubishi } \\
\text { Kyowa Hakko Kogyo } \\
\text { Burroughs Wellcome } \\
\text { BASF } \\
\text { Toyobo }\end{array}$ & $\begin{array}{l}\text { Préclinique } \\
\text { Préclinique } \\
\text { Préclinique } \\
\text { Préclinique } \\
\text { Préclinique } \\
\text { Préclinique } \\
\text { Phase III } \\
\\
\text { Préclinique } \\
\text { Phase II }\end{array}$ \\
\hline Pro-urokinase & $\begin{array}{l}\text { Mochida } \\
\text { Collaborative Research }\end{array}$ & Sandoz & $\begin{array}{l}\text { Phase I } \\
\text { Phase I }\end{array}$ \\
\hline Protéine C & $\begin{array}{l}\text { Integrated Genetics } \\
\text { Eli Lilly }\end{array}$ & Knoll/BASF & Phase I \\
\hline Superoxyde dismutase & $\begin{array}{l}\text { Biotechnology General } \\
\text { Chiron/Pharmacia } \\
\text { Syn-Tek }\end{array}$ & & $\begin{array}{l}\text { Phase I } \\
\text { Phase I }\end{array}$ \\
\hline Facteur VIII** & $\begin{array}{l}\text { Biogen } \\
\text { Chiron } \\
\text { Genetics Institute } \\
\text { Trangène }\end{array}$ & $\begin{array}{l}\text { Smith Kline Beckman } \\
\text { Teijin } \\
\text { Kabi-Vitrum } \\
\text { Nordisk } \\
\text { Baxter-Travenol } \\
\text { CNTS }\end{array}$ & \\
\hline Antithrombine III & Transgène & & Phase I \\
\hline $\begin{array}{l}\text { Atrial natriuretic } \\
\text { factor (ANF) }\end{array}$ & $\begin{array}{l}\text { California Biotechno } \\
\text { Merck } \\
\text { Monsanto }\end{array}$ & Wyeth & Phase II \\
\hline
\end{tabular}

* tPA : activateur tissulaire du plasminogène. ** Facteur VIII : facteur anti-hémophilique A. *** Boeringer Ingelheim produit «Actilase » lancé en Nouvelle-Zélande en juin 1987; Genentech « Activase » accepté le 13 novembre 1987. 


\section{REFEERENCES}

1. Hardford S. Genetic engineering and the pharmaceutical industry. Can J Pharmacol 1985 ; 118 : 467-70.

2. Milstein C, Caillo AC. Hybrid hybridomas and their use in immunochemistry. Nature 1983 ; 305 : 537-40.

3. Chapple JC, Dale R, Evans BG. The new genetics will pay its way. Lancet $1987 ; 23$ : 1189-92.

4. Market for recombinant cardiovascular proteins. Genet Technol News 1986; 6 ; 7 .

5. Genentech falls at the last fence in race to market heart drug. Nature 1987 ; 237 : 450.

6. Marlar RA, Kleiss AJ, Griffin JH. Human protein $\mathrm{C}$ : inactivation of factors $\mathrm{V}$ and VIII in plasma by the activated molecule. Ann NY Acad Sci 1981 ; 370 : 303-10.

7. Neidleman P, Currie MG, Geller DM, Cole BR, Adams SP. Atriopeptines : potential mediators of an endocrin relation ship between heart and kidney. Trends Pharmacol Sci 1984 ; 505-9.

8. Horiochi N, Holick MF, Potts JT, Rosenblatt M. A parathyroid hormone inhibitor: design and biological evaluation of a hormone analogue. Science 1983 ; 220 : 1053-116.

9. Perry RS. Tissue type plasminogen activator. Drugs Future 1985; 10 : 835-6.

10. Klausner A. Delivery becoming key for biotech drugs. Biotechnology 1986 ; 4 : 755-6.

11. Marshall GR. Computer aided drug design. Annu Rev Pharmacol Toxicol 1987; 27 : 193-213.

12. Veber DF, Freidinger RM, Perlow DS, et al. A potent hexapeptide analogue of somatostatin. Nature 1981 ; 292 : 55-8.

13. Evans BE, Rittle KE, Bock MG, et al. Design of nonpeptidal ligands for a peptide receptor : cholecystokinin antagonists. J Med Chem 1987; 30 : 1229-39.

14. Hopwood DA, Malpartida F, Kieser HM et al. Production of hybrid antibiotics by genetic engineering. Nature 1985 ; 314 : 6424.

15. Anderson S, Marks CB, Lazanus R, et al. Production of 2-keto-L-gulonate, an intermediate in L-ascorbate synthesis by a genetically modified Erwinia herbicola. Science 1985; $230: 144-9$

16. Dibner MD, Timmermans PBM. Biotechnology and the pharmaceutical industry. Hypertension 1986; 8 : 965-9.

17. Barnard EA. New technologies in neurobiology and their potential for future drug design. The World biotech report. LondonNew York: Online Publications, 1987: 113 7. sence de la fibrine qui joue le rôle de co-facteur. Cette spécificité augmentée devrait permettre une therapeutique thrombolytique efficace avec une réduction des effets secondaires, notamment hémorragiques. Le marché prévisible du tPA est très important (500 à 800 millions de dollars aux Etats-Unis) [4], ce qui a provoqué une intense compétition entre différentes sociétés pharmaceutiques. La position de leader est actuellement occupée par Genentech qui, malgré un retard inattendu à cause de la non-acceptation du dossier tPA par la food and drug administration (FDA) [5], prévoit son lancement aux États-Unis vers la fin 1987 ou au début de 1988. D'autres activateurs du plasminogène ayant une spécificité similaire, telle que la pro-urokinase (single chain urinary plasminogen activator, scuPA) sont également en cours d'évaluation clinique.

Une autre protéine ayant une activité anti-thrombolytique potentielle est la protéine $C$, facteur anticoagulant endogène qui inactive les facteurs de coagulation $\mathrm{Va}^{*}$ et VIIIa* [6]. La protéine C pourrait être utilisée seule ou en combinaison avec des activateurs du plasminogène et pourrait aussi représenter un marché considérable (environ 200 millions de dollars au début des années 1990) [4].

Un important problème lors du traitement thrombolytique est la morbidité et la mortalité engendrées par les dommages tissulaires provoqués par la formation de radicaux libres lors de la reperfusion post-ischémique. Les radicaux libres ainsi produits pourraient être neutralisés par la superoxyde-dismutase (SOD). Le gène codant pour la SOD humaine est cloné et l'enzyme est aujourd'hui proposée par plusieurs sociétés. Comme pour la protéine $C$, des ventes significatives de cette enzyme sont prévues par certains analystes (environ 200 millions de dollars dans les années 1990 [4]).

A l'autre extrémité de ces problèmes d'hémostase se trouve la coagulation défectueuse caractéristique de l'hémophilie. Comme il a été remarqué précédemment, le facteur VIII a été l'un des premiers produits identifiés comme une cible de production par la technologie de l'ADN recombinant. Malgré des difficultés techniques majeures liées à la complexité de la molécule du facteur VIII, un progrès significatif a été réalisé grâce à l'expression de cette protéine dans des cellules animales recombinantes, seules capables d'effectuer toutes les modifications post-traductionelles (glycosylation, etc.) nécessaires à la production d'un facteur VIII actif. La voie devient donc ouverte à la production d'un facteur VIII actif plus pur, de qualité reproductible et, surtout, dépourvu du risque de contamination par des virus transmis avec les dérivés sanguins.

Toujours dans le domaine cardiovasculaire, un nouveau facteur hypotenseur, le facteur atrial natriurétique, est également en cours d'évaluation clinique. Cette protéine courte $(25$ à 28 acides aminés) est elle aussi susceptible de faire l'objet d'un important marché, chiffré en centaines de millions de dollars [4, 7].

A côté du domaine cardiovasculaire, la première génération de produits issus de la biotechnoloie a déjà commencé à avoir un impact sur la pharmacopée de l'avenir dans bien d'autres domaines thérapeutiques (cancer, immunologie, inflammation, etc.).

Produits de deuxième génération. La deuxième génération de produits biotechnologiques concerne les protéines modifiées, dérivées de molécules naturelles de première génération, mais qui n'existent pas dans la nature. Les modifications apportées permettent la conservation (ou idéalement l'amélioration) de l'activité biologique souhaitée, l'amélioration d'autres propriétés pharmaceutiques telles que la stabilité in vivo, la biodisponibilité, la spécificité (et donc le profil des effets secondaires) et éventuellement celles d'autres propriétés galéni- 
ques* $^{*}$. Introduire ce type de modification implique une connaissance approfondie du mécanisme d'action et du rapport structure/fonction des biomolécules concernées.

Le prototype de cette approche de création de peptides et de protéines analogues est peut-être le travail de Horiachi et al. [8] sur les rapports structure/fonction de l'hormone parathyroïdienne (PTH). Dans leurs études, les auteurs ont montré que l'activité biologique de l'hormone naturelle de 84 acides aminés est localisée dans un fragment N-terminal de 34 acides aminés. Ce fragment $\mathrm{N}$-terminal a pu être lui-même scindé en deux sousfragments, l'un responsable de l'affinité pour le récepteur, l'autre possédant l'activité effectrice. Le fragment portant le site de liaison au récepteur entre en compétition avec l'hormone naturelle pour le récepteur à la PTH et se comporte comme un antagoniste puissant de son activité. En poussant cette approche plus loin, de nouveaux agonistes et antagonistes de la PTH ont été découverts [8].

Dans le cas des grandes protéines, une telle voie est manifestement plus difficile et accessible uniquement par génie génétique. Ainsi on peut ajouter ou enlever un domaine structurel entier ou, par mutagenèse dirigée, changer ponctuellement et systématiquement certains acides aminés. Ces techniques ont été appliquées à un grand nombre de protéines y compris les interférons, les interleukines et les facteurs de croissance. L'exemple le plus frappant et le plus important sur le plan commercial est, peut-être, la création d'un nombre important de molécules de tPA de deuxième génération. Si le tPA offre des avantages dans le traitement thrombolytique en raison de sa spécificité et de l'augmentation de son activité par la fibrine, il n'est pas sans inconvénients. Ainsi le

\footnotetext{
* Galénique : ce qui concerne la formulation et la présentation des médicaments sous forme utilisable en clinique.
}

$\mathrm{m} / \mathrm{s} n^{\circ} 4$ vol. 4, avril 88
tPA naturel possède une demi-vie très courte (1 à 4 minutes) et est relativement facilement inactivé par des inhibiteurs circulants. Plusieurs sociétés pharmaceutiques ont créé des versions modifiées de la molécule tPA avec, comme objectif, la production d'une enzyme avec une affinité plus forte pour la fibrine, une demi-vie prolongée et une moindre susceptibilité aux inhibiteurs endogènes [9]. Ce sujet est discuté plus en détail dans l'article du professeur Samama (page 222).

Le développement des produits de deuxième génération résoud également l'un des problèmes fondamentaux associés au développement et à l'exploitation des peptides et des protéines comme médicaments, celui de la brevetabilité des formes moléculaires qui existent dans la nature. Les produits de deuxième génération n'existent pas à l'état naturel. Ils peuvent donc, en principe, être protégés par des brevets, exactement comme n'importe quelle autre nouvelle entité chimique.

Produits de troisième génération. Si les produits de deuxième génération peuvent faire l'objet d'une protection industrielle, d'autres contraintes persistent quant au développement de ces molécules comme médicaments majeurs, car les peptides et les protéines qu'ils sont restent difficiles à utiliser en thérapeutique. De fait, la plupart des drogues majeures sont des molécules organiques de bas poids moléculaire. En tant que telles, ces dernières peuvent généralement être administrées par voie orale et elles peuvent agir tant au niveau extracellulaire qu'intracellulaire et ne provoquer normalement aucune réponse immunitaire.

De façon générale, protéines et peptides répondent mal à ces critères. Ce sont des molécules de poids moléculaire élevé, facilement dégradables par les protéases et difficilement transportables dans l'organisme. En conséquence, il est nécessaire de les administrer par voie parentérale. En général, l'organisme développe une réponse immuni- taire contre ces protéines exogènes et ceci est également le cas lorsque des doses massives d'une protéine normalement présente dans l'organisme, mais à concentration infime, sont administrées. Bien que des progrès importants aient été faits, notamment dans la technologie mise au point par California Biotechnology pour l'administration des protéines et des peptides à travers des surfaces muqueuses [10], ces problèmes restent un obstacle fondamental au développement des protéines en tant que médicaments de premier ordre. C'est pour cette raison qu'à moyen terme, beaucoup d'espoir repose sur la création d'une troisième génération de produits pour laquelle tout l'arsenal de la biotechnologie sera utilisé pour découvrir ou pour produire des molécules organiques originales qui répondront aux critères cités ci-dessus.

La voie la plus directe (et aussi la plus complexe et la plus sophistiquée) par laquelle l'approche biotechnologique pourrait amener au développement de telles molécules, utilise la chimie organique synthétique pour mimer la structure tridimensionnelle des régions actives des protéines et des peptides, structure connue grâce à l'étude des produits de première et de deuxième génération. Cette approche pourrait être appliquée à l'étude de l'interaction des enzymes avec leurs inhibiteurs, leurs substrats ou leurs co-facteurs spécifiques, ou de l'interaction entre des récepteurs et leurs ligands polypeptidiques tels que neuropeptides, facteurs de croissance, lymphokines ou hormones.

Une analyse extrêmement détaillée de la nature physico-chimique de ces interactions est nécessaire pour permettre une approche de modélisation (drug design). Cette analyse est principalement déterminée par les données disponibles sur la structure tridimensionnelle de la cible moléculaire thérapeutique. L'augmentation de la puissance informatique disponible, de l'amélioration des détecteurs de rayons $\mathrm{X}$, et l'application des 
techniques de résonance magnétique nucléaire (RMN) sur les macromolécules non cristallines rendent cette analyse techniquement réalisable. La biotechnologie assure l'isolement, le clonage et l'expression du gène codant pour la molécule cible, la rendant disponible à l'état pur et en quantité suffisante pour permettre l'application de ces techniques [11].

Ce type d'approche, bien que non encore appliqué à des grandes protéines, a été utilisé dans le développement des analogues de la somatostatine, un peptide de 14 acides aminés. La molecule naturelle possède tous les inconvénients classiques d'un peptide, étant inactif par voie orale et possédant une demi-vie très courte. Veber $e t$ al. [12] ont démontré que seulement quatre des 14 acides aminés sont directement impliqués dans l'interaction avec le récepteur, les autres étant nécessaires pour présenter ces quatre acides aminés dans la configuration spatiale correcte. Un analogue de six acides aminés a été construit et cette molécule, résistante aux protéases, garde son activité biologique et est active par voie orale [12]. Un raisonnement similaire a été appliqué à la conception des inhibiteurs de plusieurs enzymes ayant une importance clinique, telles que l'enzyme de conversion (ACE), la rénine et l'enképhalinase.

Il y a encore très peu de temps, seuls des analogues peptidiques ont été conçus pour mimer les ligands peptidiques naturels. Les travaux récents de Evans $e t$ al. [13] concernant le développement des antagonistes au neuropeptide cholécystokinine, ont montré clairement que la production d'analogues spécifiques entièrement non peptidiques et oralement actifs est réalisable.

\section{De nouvelles cibles thérapeutiques}

Une autre façon par laquelle la nouvelle biotechnologie peut permettre et faciliter le développe- est la fourniture d'outils permettant une compréhension des mécanismes moléculaires en cause dans une maladie. Des cibles moléculaires et des facteurs endogènes connus ou soupçonnés d'exister en quantités infimes peuvent maintenant être rapidement isolés, clonés, exprimés et leur fonction biologique évaluée. Une fois que l'on sait quelles protéines sont impliquées dans un processus de contrôle biologique ou quelles protéines sont modifiées qualitativement ou quantitativement dans des états pathologiques, des nouvelles cibles sont immédiatement suggérées. Ces cibles peuvent ensuite
Cette approche permet l'étude du récepteur dans des conditions définies et donc une évaluation plus précise de ses caractéristiques pharmacologiques.

Evidemment, l'application de la technique de mutagenèse dirigée à un tel récepteur, ou l'utilisation d'anticorps monoclonaux antipeptides, pourrait également aider à la définition des propriétés fonctionnelles et topologiques du récepteur. Ceci pourrait permettre la conception des molécules non peptidiques analogues aux neurotransmetteurs connus.

Une autre façon par laquelle la biotechnologie peut aider à l'identification de nouveaux

Tableau IV

PROTÉINES RÉCEMMENT RECONNUES COMME POSSÉDANT DES FONCTIONS SPÉCIFIQUES DANS LES TISSUS NERVEUX

- Récepteurs des neurotransmetteurs et des hormones

- Protéines des canaux ioniques

- Protéines de traduction des signaux associés aux récepteurs et aux canaux ioniques

- Protéines précurseurs des neuropeptides modulateurs

- Protéines impliquées dans la libération et le transport des neurotransmetteurs

- Facteurs de croissance spécifiques des neurones

- Facteurs d'adhésion et de reconnaissance neuronale

\section{(Adapté d'après [17]).}

être soumises à des investigations pharmacologiques.

Comme illustration de ces principes dans le domaine de la neurobiologie, le Tableau IV indique plusieurs protéines qui ont été relativement récemment caractérisées et possèdent des fonctions spécialisées dans les tissus nerveux, représentant donc de nouvelles cibles potentielles pour une intervention pharmacologique. Une telle approche completerait l'approche existante orientée presque exclusivement vers les récepteurs. Pour donner un exemple précis, il est maintenant possible d'étudier en détail des neurorécepteurs purs après clonage du gène et expression de celui-ci dans une cellule non neuronale. outils pour la recherche pharmacologique est l'analyse génétique moléculaire des maladies. Cette approche peut révéler le mécanisme pathologique d'une maladie et, là encore, suggérer de nouvelles cibles thérapeutiques.

\section{Biotechnologie et production de petites molécules}

Enfin, la biotechnologie peut aussi contribuer au développement de nouveaux médicaments en fournissant une aide directe à la chimie organique pour la synthèse des petites molécules. Historiquement, comme il l'a été mentionné au début de cet article, beaucoup de molécules possédant 
une activité pharmacologique (tels les antibiotiques et les vitamines) ont toujours été produites par des procédés de fermentation. De tels procédés impliquent souvent des chemins de biosynthèse très complexes, dont la vitesse peut être limitée par une ou plusieurs enzymes. La nouvelle biotechnologie peut améliorer ces procédés par clonage du gène codant pour l'enzyme de l'étape limitante, éliminant ainsi un goulot d'étranglement dans la production. Pour améliorer les rendements d'une façon significative, il pourrait être nécessaire de cloner successivement plusieurs enzymes du chemin biosynthétique.

Des antibiotiques entièrement nouveaux ont également été créés; l'accessibilité des gènes de synthèse des antibiotiques permet une nouvelle approche à la recherche des antibiotiques originaux, recherche orientée jusqu'à présent vers la mutagenèse ou vers la fusion inter-spécifique des micro-organismes producteurs. Ainsi, Hopwood et al. [14] ont transféré le cluster (c'est-à-dire le groupe) des gènes contrôlant la biosynthèse de l'antibiotique isochromanequinoïde actinorhodin dans Streptomyces coelicolor, à d'autres espèces de Streptomyces productrices d'autres antibiotiques de la même classe. A la suite de cette manipulation, la synthèse de plusieurs antibiotiques complètement nouveaux a été observée [14].

De nouvelles voies de synthèse pour des molécules déjà connues peuvent aussi être conçues. En 1985, Anderson et al. ont révolutionné la synthèse de vitamine $C$ en remplaçant le procédé de production conventionnelle (une étape microbiologique et quatre étapes chimiques) par un nouveau procédé qui utilise un seul gène de Corynebacterium inséré dans le génome de Erwinia [15]. La combinaison des enzymes endogènes de Erwinia et de l'enzyme ajoutée de Corynebacterium catalyse la production directe de l'acide 2-céto-L-gulonique à partir de glucose. Il reste à faire une seule étape chimique pour la conversion de ce produit en acide ascorbique.

Un travail considérable se poursuit pour diminuer les coûts de la production en masse de ce type de produits et pour découvrir de nouveaux systèmes enzymatiques capables d'accomplir des conversions chimiques originales. Dans le cas d'une synthèse stéréospécifique très difficile, pour laquelle des enzymes avec la spécificité requise ne sont pas disponibles, il pourrait être possible d'utiliser les techniques de protein engineering (ingéniérie des protéines) afin de modifier les propriétés des enzymes existantes et de fournir des réactifs enzymatiques originaux au chimiste organicien.

Dans cet article, nous avons discuté comment l'approche par le génie génétique et les biotechnologies peuvent révolutionner les industries de la santé dans les domaines diagnostique, prophylactique et thérapeutique.

De nouveaux produits ont déjà été développés dans tous ces domaines et un apport majeur est attendu de cette approche dans la conception de nouveaux types de médicaments basée sur l'étude des cibles thérapeutiques originales.

Malgré tous ces progrès, la nouvelle biotechnologie est toujours dans son enfance et des avancées techniques majeures, et donc des améliorations de produits actuellement identifiés, peuvent être attendues à court terme. L'application de cette technologie à l'industrie pharmaceutique et son incorporation dans une stratégie pharmacologique globale sont encore plus nouvelles. Des synergies non encore identifiées entre ces deux domaines promettent des percées importantes.

L'impact de la biotechnologie sur l'industrie pharmaceutique, est d'ores et déjà énorme, à la fois conceptuellement et commercialement, bouleversant globalement la stratégie pharmacologique. Nous pouvons prédire avec confiance que cet impact continuera à se développer et à s'élargir dans les années à venir

\section{Summary}

Modern biotechnology and genetic engineering techniques will have a major conceptual and commercial impact on all aspects of health care: diagnostic, prophylactic and therapeutic. Already the newly acquired capacity to produce large quantities of pure recombinant proteins has resulted in a first generation of products such as vaccines, interferons and tissue plasminogen activator. «Second generation » products, in the form of novel derivatives of these natural proteins, are already far advanced in clinical trials. The stage is set for the utilisation of computer graphics and modelling techniques to design new potential drugs on the basis of structure function relationships derived from study of first and second generation products. In addition, biotechnology and genetic engineering will make major contributions to the drug discovery process by permitting access, to new enzyme and receptors, and their mediators and affectors which may represent novel molecular targets for pharmacological research. Finally, direct application of this new technology to chemical and biochemical production processus may radically alter the methods available for the synthesis of known drugs.

\section{TIRÉS A PART}

T. Cartwright: Recherche Biotechnologique Rhône-Poulenc Santé, Centre de Recherche de Monts, 18 rue de Montbazon, 37260 Monts, France. 